\title{
Improvement of chemotherapy induced colitis with serum-derived bovine immunoglobulin
}

\author{
Gerald L Klein ${ }^{1,2 *}$, Eric M Weaver ${ }^{2}$ \\ From Metabolism, diet and disease \\ Washington, DC, USA. 29-31 May 2012
}

\section{Background}

Chemotherapy-induced diarrhea is a common problem, with pain, ulceration, bloating, vomiting and diarrhea occurring in about $40 \%$ of patients on a standard regimen and dose [1]. The pathophysiology of this problem is quite complex, involving multiple pathways including increases in pro-inflammatory mediators tumor necrosis factor- alpha (TNF- $\alpha$ ) and interleukin-6 (IL-6) [2]. Serum-derived bovine immunoglobulin (SBI) has been used for years; first as a valued feed ingredient for weaned pigs where it has been shown to decrease mortality and morbidity and increase growth rates [3]; and then as a safe dietary supplement in humans [4]. In experimental animal models, SBI has been found to decrease both TNF $-\alpha$ and 1 L- 6 in intestinal inflammation, and decrease intestinal permeability [5].

\section{Case history}

This patient is a white female, 54 years of age, who developed a renal cell carcinoma and an unrelated adenocarcinoma of the ovary. She underwent a nephrectomy, empiric lymph node dissection, omentectomy, and bilateral salpingo-oophrectomy. A 6-week course of carboplatin and paclitaxel was prescribed for the treatment of the carcinoma of the ovary. Consistent with previous reports, this treatment regimen induced abdominal pain, nausea, vomiting, and diarrhea; resulting in a 12 kilogram weight loss. With the physician's approval, she began using $2 \mathrm{~g}$ of SBI b.i.d as a food supplement.

\section{Results}

After using SBI, this patient's symptoms improved while she continued with chemotherapy. She gained 2.5 kilograms in the next 2 weeks, without side effects from SBI and was able to successfully complete her course of chemotherapy. SBI has previously been shown to decrease diarrhea and, recently, has been shown to decrease intestinal permeability [6].

\section{Conclusions}

SBI may have improved this patient's gastrointestinal complaints and maintenance of body weight. Clinical and nonclinical studies are warranted to determine the efficacy of this food product in decreasing the side effects associated with chemotherapy treatment.

\section{Author details}

${ }^{1}$ Thomas J Long School of Pharmacy and Health Science at the University of the Pacific, USA. ${ }^{2}$ Entera Health, Inc., Cary, NC, 27511, USA.

Published: 1 June 2012

\section{References}

1. Stringer AM, Gibson RJ, Logan RM, et al: Chemotherapy-induced diarrhea is associated with changes in the luminal environment in the DA rat. Exp Biol Med (Maywood) 2007, 232:96-106.

2. Bowen JM, Keefe DK: New pathways for alimentary mucositis. J Onc 2008, 1-7.

3. Peace RM, Campbell J, Polo J, et al: Spray-dried porcine plasma influences intestinal barrier function, inflammation, and diarrhea in weaned pigs. J Nutr 2011, 141:1312-1317.

4. Lembcke J, Pearson J, Brown K: Acceptability, safety, and digestibility of spray-dried bovine serum added to diets of recovering malnourished children. J Pediatr Gastroenterol Nutr 1997, 25:381-384.

5. Moretó M, Pérez-Bosgue A: Dietary plasma proteins, the intestinal immune system, and the barrier functions of the intestinal mucosa. J Anim Sci 2009, 87:E92-E100.

6. Pérez-Bosgue A, Miro L, Polo J, et al: Dietary plasma protein supplements prevent the release of mucosal proinflammatory mediators in intestinal inflammation in rats. J Nutr 2010, 140:25-30.

doi:10.1186/1753-6561-6-S3-P26

Cite this article as: Klein and Weaver: Improvement of chemotherapy induced colitis with serum-derived bovine immunoglobulin. BMC Proceedings 2012 6(Suppl 3):P26.

${ }^{1}$ Thomas J Long School of Pharmacy and Health Science at the University of the Pacific, USA

Full list of author information is available at the end of the article 